\title{
Pre-Clinical Evaluation of Proprietary Lutein, Zeaxanthin, and Rosemary Formulation for Its Dermal Protective Activity in Male Swiss Albino Mice
}

\author{
Huijin Heo', Jayashree Madhavan², Sangwon Eun ${ }^{3}$, Hyunmook Jung ${ }^{4}$, and Hana Lee ${ }^{1}$ \\ ${ }^{1}$ Department of Food Science and Biotechnology, Chungbuk National University, Chungbuk 28644, Korea \\ ${ }^{2}$ Katra Phytochem Pvt. Ltd., Bangalore 560008, India \\ ${ }^{3}$ Daehan Chemtech Co., Ltd., Seoul 01811, Korea \\ ${ }^{4}$ Cosmax Bio, Inc., Chungbuk 28644, Korea
}

\begin{abstract}
This study aimed to evaluate the efficacy of the proprietary lutein, zeaxanthin, and rosemary formulation for its dermal protection against ultraviolet (UV) irradiated skin dehydration. A total of 48 male Swiss albino mice of $8 \sim 12$ weeks of age were divided into eight groups of 6 mice: mice in group 1 (G1) were considered the normal control, without treatment and without skin shaving; mice in G2 had their skins were shaved, but did not receive treatment; mice in G3 were the pathological control; mice in G4 were treated as standard (hyaluronic acid); mice in G5 G8 were treated with low and high doses of 2 different test substances, respectively. Mice were anaesthetized and then depilatory was applied on the dorsal skin area $(2 \mathrm{~cm} \times 2 \mathrm{~cm})$ on alternate days, then UV/blue light irradiation was carried out for 15 min for 6 weeks. Collagen type 1 gene expression was determined via densitometric analysis, skin elasticity was assessed, and stratum corneum water contents were measured using a cutometer and corneometer. Skin hydration was assessed through transepidermal water loss, and several serum biochemical parameters (collagenase, hydroxyproline, hyaluronic acid, and ceramide levels) were determined to assess the skin moisturizing activity of the product. Images for assessing photoaging were considered between different groups on day 42 . All these subjective parameters reached statistical significance $(P<$ 0.05 ) in groups treated with the proprietary lutein and rosemary formulation compared with the placebo-treated group. In conclusion, the proprietary lutein, zeaxanthin, and rosemary formulation showed better protection of skin subjected to UV irradiated skin dehydration.
\end{abstract}

Keywords: dermal protection, moisturizer, skin irradiation, transepidermal water loss

\section{INTRODUCTION}

Solar ultraviolet (UV) irradiation penetrates the skin causing sunburn, mild inflammation, photocarcinogenesis, and premature skin aging (Cooper et al., 1992). Photo-aging is the most common form of skin damage (due to increase collagen degradation, irregular pigmentation, and wrinkles) caused by chronic, repetitive sun exposure (Fisher et al., 1997). Long-term exposure to solar UV irradiation damages the dermal connective tissue and extracellular matrix (ECM), which in turn leads to aged appearance of photo-damaged skin (Pittayapruek et al., 2016). A hallmark of this UV-induced ECM remodeling is degradation of collagen and elastin through UV-induced activation of matrix metalloproteinases and decreased de novo synthesis of collagen (Quan et al., 2009). The mechanisms of UV-induced matrix metalloproteinase activation and inhibition of collagen synthesis have been studied in detail (Fisher et al., 1996). In contrast, the effect of UV on hyaluronic acid (HA), another key component of the dermal ECM, is much less understood, and the underlying mechanisms are primarily unknown. HA is a linear polymer composed of repeating disaccharides (D-glucuronic acid- $\beta-1,3-\mathrm{N}$-acetylglucosamine- $\beta-1,4-)$ and assembled from the respective activated nucleotide sugars (uridine diphosphate-glucuronic acid, uridine diphosphate$\mathrm{N}$-acetylglucosamine) at the inner plasma membrane by HA synthases (Papakonstantinou et al., 2012). In the skin, HA also has important structural functions that are related to the unique molecular features of HA. The high 
polymer length and polyanionic charge enables HA to bind water, which in turn supports volume expansion and turgidity of the skin, diffusion of metabolites and nutrients, and elasticity of the skin (Manuskiatti and Maibach, 1996). Taken together, HA confers functions through initiating receptor signaling, and creates an extracellular microenvironment that supports the typical physicochemical and mechanical properties of the skin (Papakonstantinou et al., 2012).

In the epidermis, $\mathrm{HA}$ is induced during wound healing and regeneration (Tammi et al., 2005), whereas the regulatory pathways of HA are much less understood in the dermis. Interestingly, several conditions that accelerate skin aging, such as estrogen deficiency (Sator et al., 2004; Kanda and Watanabe, 2005), are associated with loss of HA from the dermis, and treatments that counteract actinic skin aging, such as retinoic acid, increase dermal HA (Margelin et al., 1996; Saavalainen et al., 2005). These results strongly suggest that dermal HA plays an important role during photo-aging.

Lutein and zeaxanthin are common carotenoid xanthophylls found in nature, and are especially rich in Marigold flowers (Quackenbush and Miller, 1972). Lutein and zeaxanthin are stereoisomers in the human retina and are hydroxy carotenoids demonstrating potent antioxidant activity (Roberts et al., 2009). Commonly present in the human macula and retina, these macular carotenoids are majorly studied for eye health in protecting the eye from UV photo-toxicity by quenching reactive oxygen species (ROS). Thus, they are commonly studied in age-related macular degeneration and cataract (Sommerburg et al., 1999). Because of their good antioxidant potency, lutein and zeaxanthin are known to help maintain better skin tone, skin lightening, and elasticity (Mathews-Roth and Krinsky, 1985; Roberts et al., 2009).

Furthermore, rosemary extract is a potent antioxidant (Klancnik et al., 2009). The antioxidant properties of rosemary are based on the carnosic acid and carnosol with rosemarinic acid, which accumulate in the fatty membranes of cells. Carnosic acid is one of the most potent antioxidants (Aruoma et al., 1992). The antioxidant properties of rosemary extract are attributed to its richness in isoprenoid quinones, which act as chain terminators of free radicals and as chelators of ROS (Nieto et al., 2018). In addition, carnosic acid and carnosol act as potent scavengers of peroxyl radicals (Aruoma et al., 1992). These dietary carotenoids cannot be synthesized by mammals and must therefore be obtained from diet (Jia et al., 2017). Previous research on lutein and zeaxanthin has shown that, as oral or topical treatments, these supplements induce remarkable improvements in skin elasticity, skin hydration, and skin lipid levels, contributing to anti-wrinkle and photo-protective effects (Palombo et al., 2007). One of the best commercial sources of pure lutein and zea- xanthin is marigold flowers (Jia et al., 2017). Known as a potent antioxidant, Rosmarinus officinalis has been used as a healing herb for centuries. Over the last few decades, extensive in vitro, in vivo, and human trials have been conducted to determine scientific evidence for the medicinal properties attributed to rosemary and its active constituents, including carnosic acid (Park et al., 2013; GonzálezMinero et al., 2020). Diterpene carnosic acid, one of the most important active components of rosemary extract, has been studied globally for health benefits for skin, mainly due to its capacity to prevent the generation of radical species (Loussouarn et al., 2017).

The aim of the present study was to elucidate the protective effects of a proprietary lutein, zeaxanthin, and rosemary formulation against harmful UV radiations on dermal HA, and various other parameters including collagen type 1, transepidermal water loss (TEWL), and estimation of important biochemical parameters that may play a key role in skin health.

\section{MATERIALS AND METHODS}

\section{Animals}

In-house bred male Swiss albino mice aged between 8 and 12 weeks were housed under controlled conditions: temperature of $22^{\circ} \mathrm{C} \pm 3^{\circ} \mathrm{C}$, relative humidity of $50 \%$ to $70 \%$, and a 12-h light and 12-h dark cycle. Animals were housed in standard polypropylene cages with stainless steel top grills with facilities for pelleted food and drinking water via a bottle. Sterile paddy husk was used as bedding material and was changed every day. The feed and drinking water were free from any contaminants and provided ad libitum. All the experimental procedures and protocols used were reviewed and approved by the Institutional Animal Ethics Committee (IAEC) of Radiant Research Services Pvt. Ltd. (Karnataka, India) (Regd. No.: 1803/PO/RcBi/S/2015/CPCSEA) constituted in accordance with the guidelines of the Committee for the Purpose of Control and Supervision of Experiments on Animals, government of India.

\section{Preparation of test samples}

Test sample A: This was the placebo and natural sunflower oil, which is the base carrier vehicle of the active proprietary formulation. Sunflower oil, a vegetable oil, was considered the best carrier/vehicle since it is stable and effective in this medium.

Test sample B: This was the active and proprietary Xan$\operatorname{Max}^{\circledR} 80$ (NutriScience Innovations LLC, Milford, CT, USA) and rosemary formulation (ratio: 11.11:88.88), dispersed in sunflower oil. XanMax ${ }^{\mathbb{R}} 80$ (NutriScience Innovations) is a marigold extract that contains $80 \%$ free lutein and $6 \%$ zeaxanthin. Lutein and zeaxanthin ratios 
were based on dietary reference intakes, whereby there is strong evidence that lutein is safe up to $20 \mathrm{mg} / \mathrm{d}$ (Ranard et al., 2017). Dried marigold was extracted in hexane and subjected to saponification. The extract was filtered, evaporated until dry, and frozen until use. Rosemary extract, which contained $8 \%$ carnosic acid, was extracted in ethanol and evaporated until dry. According to EU regulation $1333 / 2008$, the maximum level $(\mathrm{mg} / \mathrm{L}$ or $\mathrm{mg} / \mathrm{kg}$ ) of rosemary extracts in food supplements should be $400 \mathrm{mg} / \mathrm{kg}$. Our formulation contains less rosemary extract than the maximum allowed dose per day (de Raadt et al., 2015). During the formulation stage, the dose of individual active ingredients (lutein, zeaxanthin, and rosemary) was taken into consideration following scientifically supported dietary guidance and intake recommendations.

\section{Experimental design}

A total of 48 male mice were divided into eight groups of 6 mice (Table 1). At the start of the experiment, mice in groups (G) 2 to 8 were anesthetized on the dorsal skin surface, and then depilatory was applied to the dorsal skin $(2 \mathrm{~cm} \times 2 \mathrm{~cm})$ on alternate days. UV irradiation (equivalent to $14 \mathrm{~mJ} / \mathrm{cm}^{2}$ ) was carried out using UV simulators fitted with a UV bulbs for 15 minutes with full spectrum UV radiation (i.e., $260 \sim 400 \mathrm{~nm}$ ) five times a week during the 6 week treatment period. Subsequently, animals were anaesthetized using isoflurane anesthesia, blood samples collected via retro-orbital route for biochemical analyses. Animals were sacrificed using extended isoflurane anesthesia and skin was isolated for further biochemical evaluations.

\section{Measurement of collagen-I gene expression in skin by RT- PCR}

mRNA expression of collagen type 1 was carried out using semi-quantitative reverse transcriptase-polymerase chain reaction (RT-PCR). Briefly, control and treated frozen mice skin was crushed into a powdered form by adding liquid nitrogen and using a pestle and mortar. Total cellular RNA was isolated using Tri Reagent (Thermo Fisher Scientific, Waltham, MA, USA) according to the manufacturer's protocol. cDNA was synthesized from total isolated RNA by a reverse transcriptase kit according to manufactures instructions (Thermo Fisher Scientific). Then, $50 \mu \mathrm{L}$ of the reaction mixture was subjected to PCR for amplification of mice cDNA using specifically designed primers procured from Eurofins India. The housekeeping gene, glyceraldehyde 3-phosphate dehydrogenase (GAPDH) was co-amplified in each reaction as a control. PCR conditions included: denaturation at $95^{\circ} \mathrm{C}$ for $30 \mathrm{~s}$, annealing for $30 \mathrm{~s}$, and extension at $72^{\circ} \mathrm{C}$ for $1 \mathrm{~min}$. This was followed by a final extension at $72^{\circ} \mathrm{C}$ for $10 \mathrm{~min}$. The primers used for second strand synthesis were: forward, 5'-GTGCTAAAGGTGCCAATGGT-3'; reverse, 5'-ACC AGGTTCACCGCTGTTAC-3'. The PCR-amplified products were analyzed using $1 \%$ agarose gel electrophoresis. Gels were scanned using the Alpha Digi Doc system (Alpha Innotech, San Leandro, CA, USA) and subjected to semi-quantitative densitometric analysis using Alpha Imager software (Alpha Innotech).

\section{Measurement of TEWL}

Photographic skin exposed to UV irradiation from day 0 to 42 of the treatment period was assessed by TEWL, detected using a Tewameter (Courage and Khazaka Electronics, Cologne, Germany). TEWL scores are digitally generated by measuring the dielectric properties of the skin. For TEWL measurements, room temperature and humidity were maintained at $21^{\circ} \mathrm{C} \pm 1^{\circ} \mathrm{C}$ and $50 \% \pm 5 \%$, respectively. The test mice were shifted to the room and allowed to stabilize for $30 \mathrm{~min}$ prior to measuring the TEWL scores. Several measurements were performed on

Table 1. Experimental groups of mice designed for pre-clinical evaluation of test substances on dermal protective activity

\begin{tabular}{|c|c|c|c|}
\hline Group & Group description & Treatment description & $\begin{array}{l}\text { No. of } \\
\text { animals }\end{array}$ \\
\hline G1 & Cell control & No treatment and no skin shaving (no UV light irradiation nor product treatment) & 6 \\
\hline $\mathrm{G} 2$ & Sham control & No treatment, but mice were shaved & 6 \\
\hline G3 & Pathological control & Mice were shaved and exposed to UV radiation, but did not receive a test product & 6 \\
\hline G4 & $\begin{array}{l}\text { Standard control } \\
\text { (hyaluronic acid } \\
120 \mathrm{mg} / \mathrm{kg} \text { ) }\end{array}$ & $\begin{array}{l}\text { Mice were shaved and exposed to UV radiation, followed by treatment with standard } \\
\text { product }\end{array}$ & 6 \\
\hline G5 & $\begin{array}{l}\text { Test A group } \\
(66.28 \mathrm{mg} / \mathrm{kg})\end{array}$ & $\begin{array}{l}\text { Mice were shaved and exposed to UV radiation, followed by treatment with } \\
\text { test product } A \text { at a low dose }\end{array}$ & 6 \\
\hline G6 & $\begin{array}{l}\text { Test A group } \\
(133.57 \mathrm{mg} / \mathrm{kg})\end{array}$ & $\begin{array}{l}\text { Mice were shaved and exposed to UV radiation, followed by treatment with } \\
\text { test product } A \text { at a high dose }\end{array}$ & 6 \\
\hline G7 & $\begin{array}{l}\text { Test B group } \\
(66.28 \mathrm{mg} / \mathrm{kg})\end{array}$ & $\begin{array}{l}\text { Mice were shaved and exposed to UV radiation, followed by treatment with } \\
\text { test product } B \text { at a low dose }\end{array}$ & 6 \\
\hline G8 & $\begin{array}{l}\text { Test B group } \\
(133.57 \mathrm{mg} / \mathrm{kg})\end{array}$ & $\begin{array}{l}\text { Mice were shaved and exposed to UV radiation, followed by treatment with } \\
\text { test product } B \text { at a high dose }\end{array}$ & 6 \\
\hline
\end{tabular}

UV, ultraviolet. 


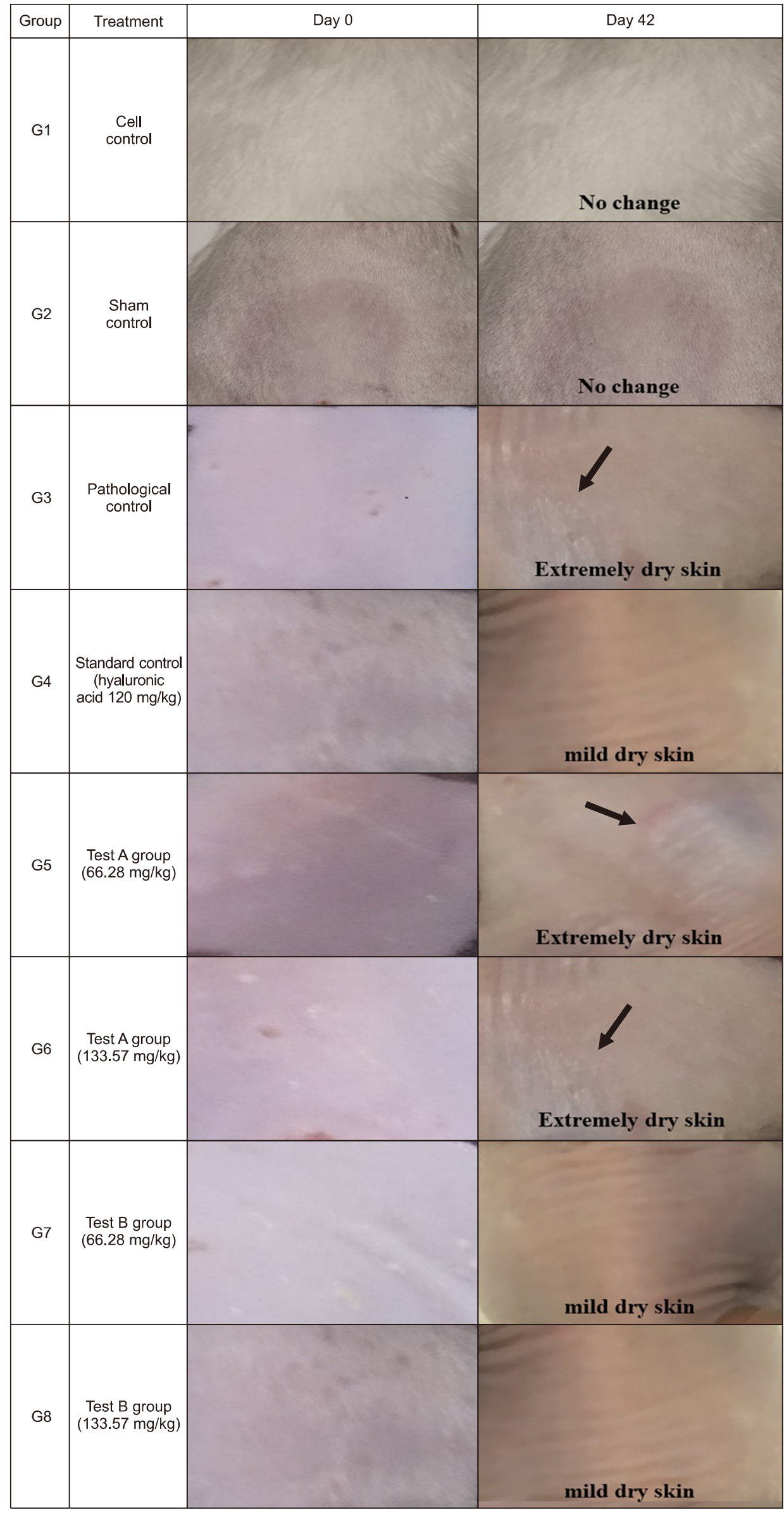

Fig. 1. Images of skin exposed ultraviolet irradiation during treatment period in experimental mice on day 0 and day 42. Skin irradiation was compared on day 0 and day 42 between group $1(\mathrm{G} 1)$ to $\mathrm{G} 8$. 


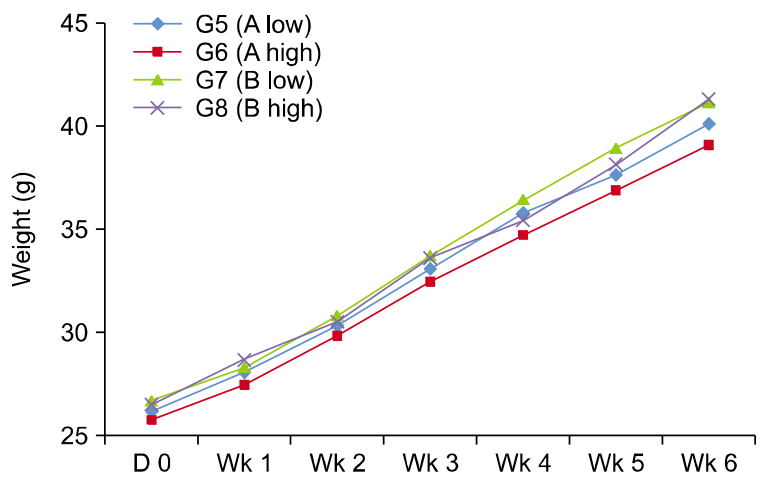

Fig. 2. Effect of test substance on body weight in mice. G5 to G8 were induced with UV-irradiation. G5 (low dose) and G6 (high dose) were treated with test compound $A ; G 7$ (low dose) and G8 (high dose) were treated with test compound B.

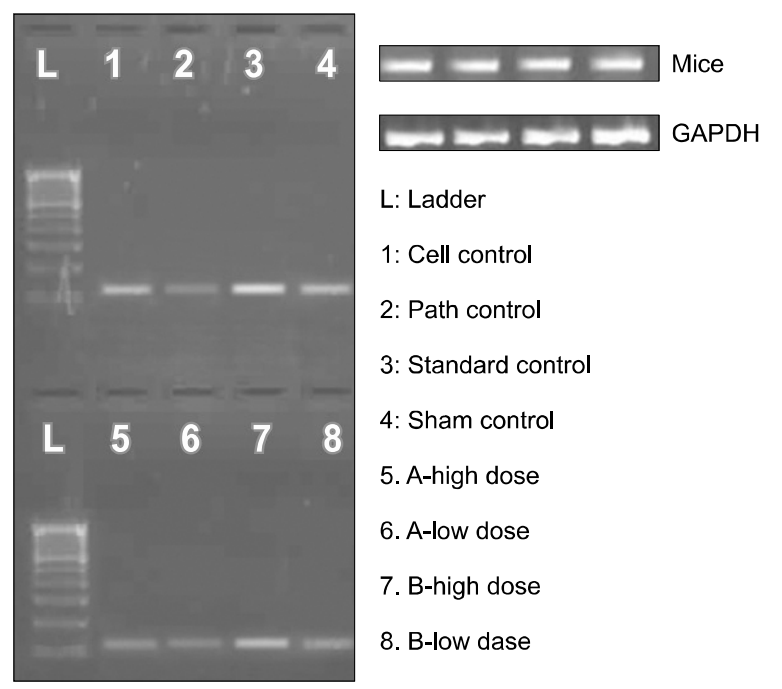

Fig. 3. Effect of test substance on mRNA expression of collagen type I in mice skin. Total RNA isolated from mice skin was used to amplify the genes. Equal quantities of cDNA was used for the reverse transcriptase-PCR reaction and equal quantities of the amplified product was used to load the wells. For normalization, glyceraldehyde 3-phosphate dehydrogenase (GAPDH) expression separately determined of the same cDNA. $L$ (protein ladder), 1 (cell control; G1), 2 (path control; G3), 3 (standard control; G4), 4 (sham control; G2) 5 (test A high dose; G6), 6 (test A low dose; G5), 7 (test B high dose; G8), and 8 (test B low dose; G7). each specimen. To avoid occlusion, the probe was not placed on the same spot twice and, after every specimen, the probe was cleaned with a soft wipe. Values were expressed as mean \pm SEM.

\section{Measurement of skin elasticity}

Photographic skin exposed to UV irradiation from day 0 to 42 of the treatment period was assessed for skin elasticity, determined using a Cutometer (Courage and Khazaka Electronics). Skin elasticity scores are digitally generated by using the suction method with the help of a Cutometer under standard conditions of temperature and humidity $\left(21^{\circ} \mathrm{C} \pm 1^{\circ} \mathrm{C}\right.$ and $\left.50 \% \pm 5 \%\right)$ and after a stabilization period of $30 \mathrm{~min}$. In principle, the suction method measures skin elevation produced by suction forces exerted over a defined area of the skin. Several measurements were performed on each specimen and the probe was not placed on the same spot twice. Values were expressed as mean $\pm \mathrm{SEM}$.

\section{Statistical analysis}

The significance of in vivo data was analyzed by one-way ANOVAs followed by Dunnett's post-hoc tests. $P<0.05$, $P<0.01$, and $P<0.001$ were considered to be statistically significant.

\section{RESULTS AND DISCUSSION}

Photographic images of UV radiation and the effects of the test products on the skin are shown in Fig. 1. Skin was not dried in G1 and G2 on Day 42 when compared to their respective Day 0 images. In G3 where pathological controls were taken, highly dried skin was observed and whereas in G4 that received hyaluronic acid, in spite of irradiation the skin was protected from dryness on Day 42. There were significant clinical changes between the skins of groups that received test samples A and B. G5 and G6 were treated with test sample A (the placebo) at low and high doses, but did not show distinct reductions in skin drying compared with G3 (the patho-

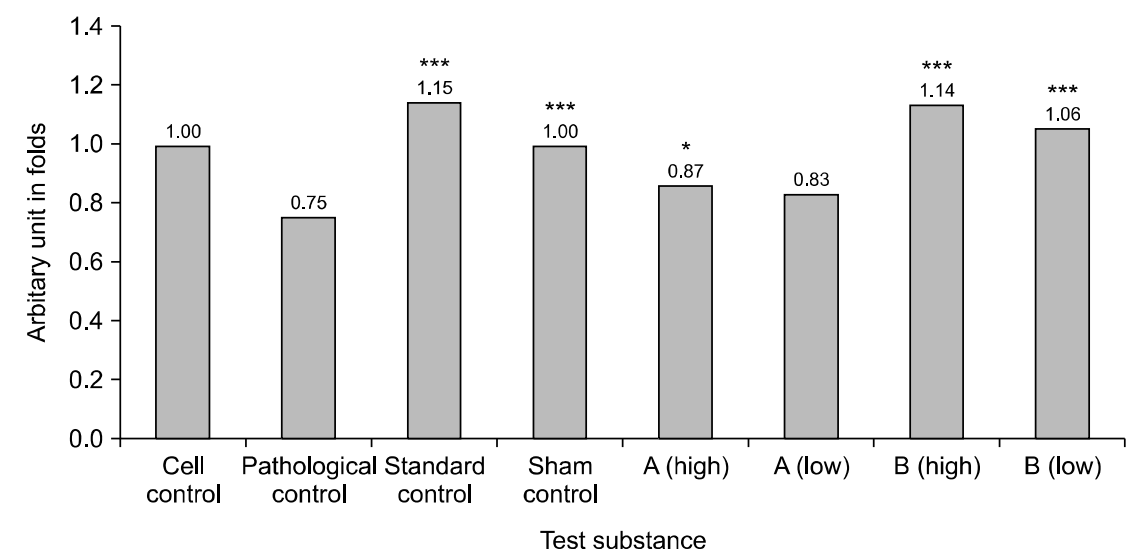

Fig. 4. Graphical representation of densitometric analysis of gene transcripts of collagen type 1 gene expression study. The density of each band is quantified by using alpha view software. The area of the peaks generated by alpha view is directly proportional to the band intensities. The fold differences between the band densities of the controls and the treatment groups were used for plotting the graph. The values were expressed in mean \pm SEM. ${ }^{*} P<0.05$ and ${ }^{* * *} P<0.001$ was considered statistically significant when compared to path control (Group 3) values. 
logical control). However, the skin of mice in G8 and G7 was protected from UV irradiation by day 42 at both high and low doses, showing good efficacy of test sample B. The body weights increased in general across all the study groups over a period of 6 weeks (Fig. 2). However, there were no clinically significant changes in the

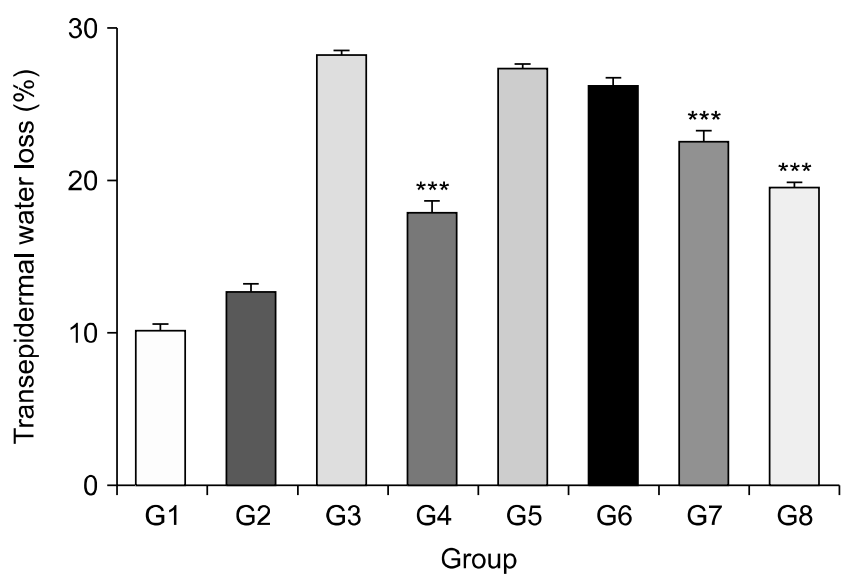

Fig. 5. Effect of test substances on transepidermal water loss (TEWL). Eight groups $(G 1 \sim G 8)$ in total were employed for the experiment. G1 was control and G2 was sham control. G3 G8 were induced with ultraviolet-irradiation; G3 was path control (no treatment). G4 was standard drug (hyaluronic acid) treated. G5 (low dose) and G6 (high dose) were treated with test compound A; G7 (low dose) and G8 (high dose) were treated with test compound B. After 42 days of treatment the TEWL score was measured using corneometer. The TEWL score is digitally generated by measuring the dielectric properties of the skin. The values were expressed in mean \pm SEM. ${ }^{* * *} p<0.001$ was considered statistically significant when compared to group 3 values.

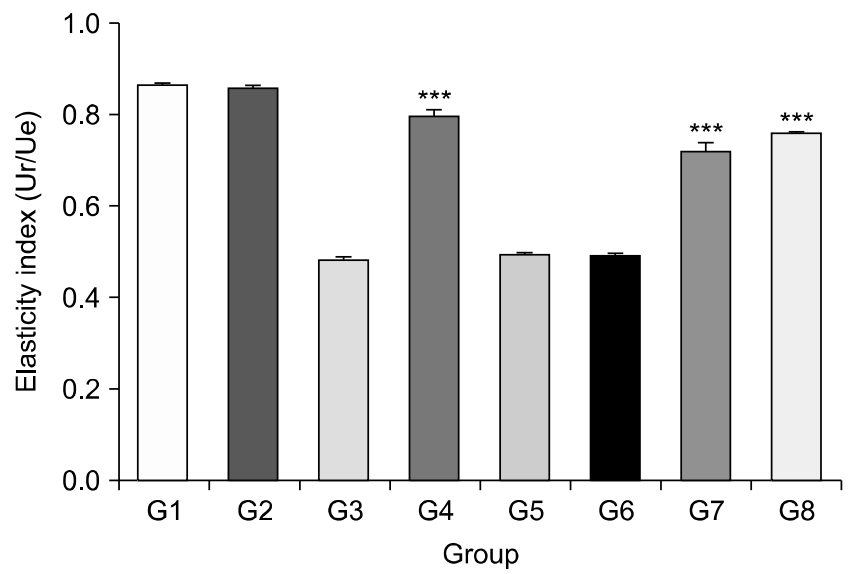

Fig. 6. Effect of test substances on skin elasticity. Eight groups $(G 1 \sim G 8)$ in total were employed for the experiment. $G 1$ was control and G2 was sham control. G3 G8 were induced with ultraviolet-irradiation; $\mathrm{G} 3$ was path control (no treatment). G4 was standard drug (hyaluronic acid) treated. G5 (low dose) and G6 (high dose) were treated with test compound A; G7 (low dose) and G8 (high dose) were treated with test compound B. After 42 days of treatment the skin elasticity score was measured using corneometer. The skin elasticity score is digitally generated by measuring the dielectric properties of the skin. The values were expressed in mean \pm SEM. ${ }^{* * *} p<0.001$ was considered statistically significant when compared to group 3 values. body weights between the 8 groups when compared with respective normal control animals on Day 0 to week 6 (Day 42) suggesting that the test products (either low or high doses) had no effects on the body weight of study animals.

To further evaluate the effects of oral intake of proprietary lutein, zeaxanthin, and rosemary formulation on skin, changes in expression of collagen type 1, TEWL, and the water contents in the stratum corneum were measured after 6 weeks of UV irradiation. The amount of collagen (Fig. 3 and 4) in the pathological control group was significantly lowered (clinically) compared to mice groups that did not receive radiation. Mice exposed to high doses of treatment B expressed collagen at similar levels to standard control (HA) mice, confirming the efficacy of test sample B at a high dose. The level of TEWL was significantly higher in mice exposed to UV light when this level was compared with that of groups that did not receive radiation. In mice that received standard treatment, TEWL levels were lower, similar to normal. Also, the group treated with test sample B showed levels of TEWL lowered in a dose-dependent manner (Fig. 5). TEWL was significantly increased in G3 compared with normal and sham control groups, and was significantly reduced in G7 and G8 compared with G3 (each $P<0.001$ ). In addition, skin elasticity (Fig. 6), water contents in the stratum corneum (Fig. 7), and all the 4 biochemical parameters (HA, ceramide, hydroxyproline, and collagenase; Fig. 8) showed opposite patterns to TEWL in mice that received UV radiation.

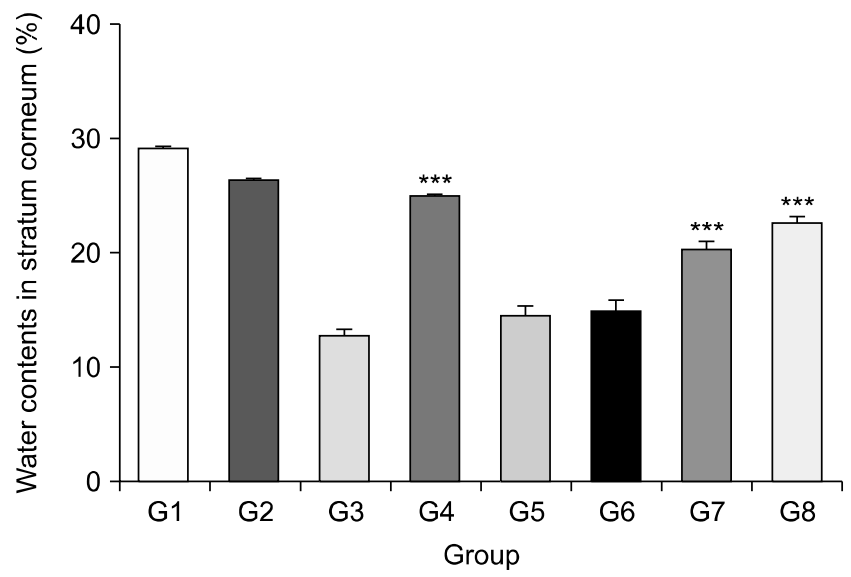

Fig. 7. Effect of test substances on water contents in stratum corneum. Eight groups $(\mathrm{G} 1 \sim \mathrm{G} 8)$ in total were employed for the experiment. G1 was control and G2 was sham control. G3 G8 were induced with ultraviolet-irradiation; G4 was standard drug (hyaluronic acid) treated. G5 (low dose) and G6 (high dose) were treated with test compound A; G7 (low dose) and G8 (high dose) were treated with test compound B. After 42 days of treatment the water contents in stratum corneum were measured using corneometer. The water contents in stratum corneum were digitally generated by measuring the dielectric properties of the skin. The values were expressed in mean \pm SEM. ${ }^{* * *} P<0.001$ was considered statistically significant when compared to group 3 values. 
A
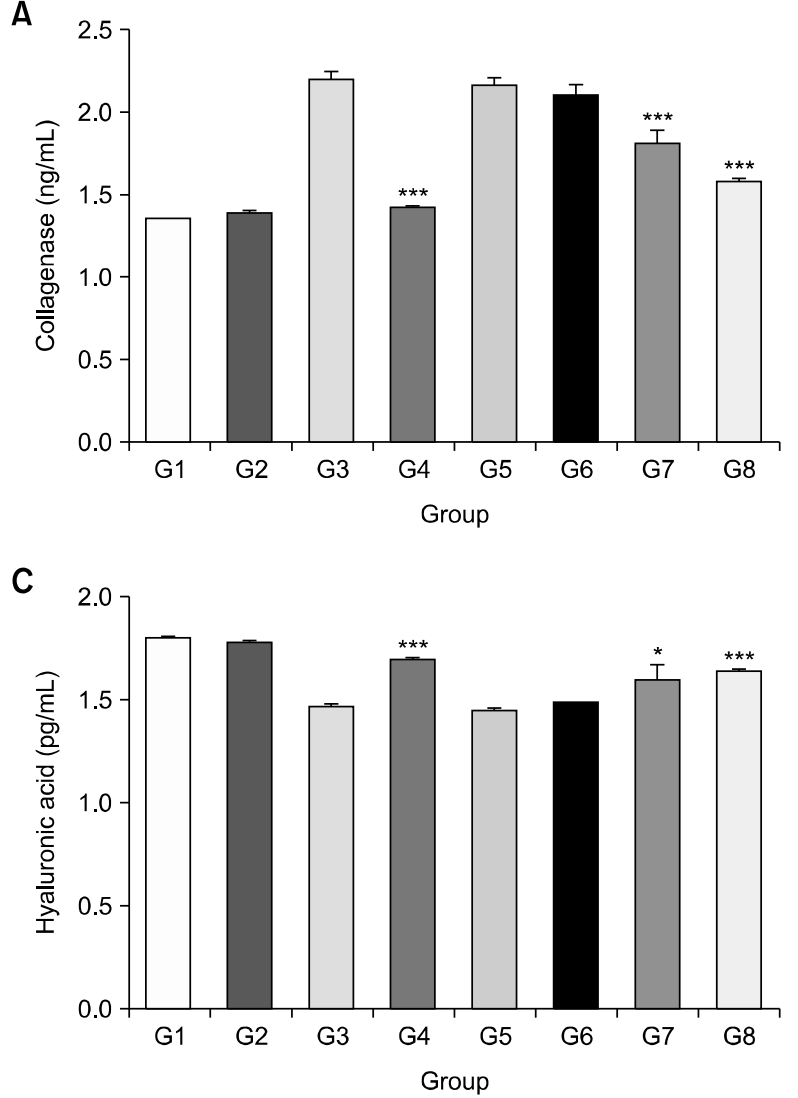

B
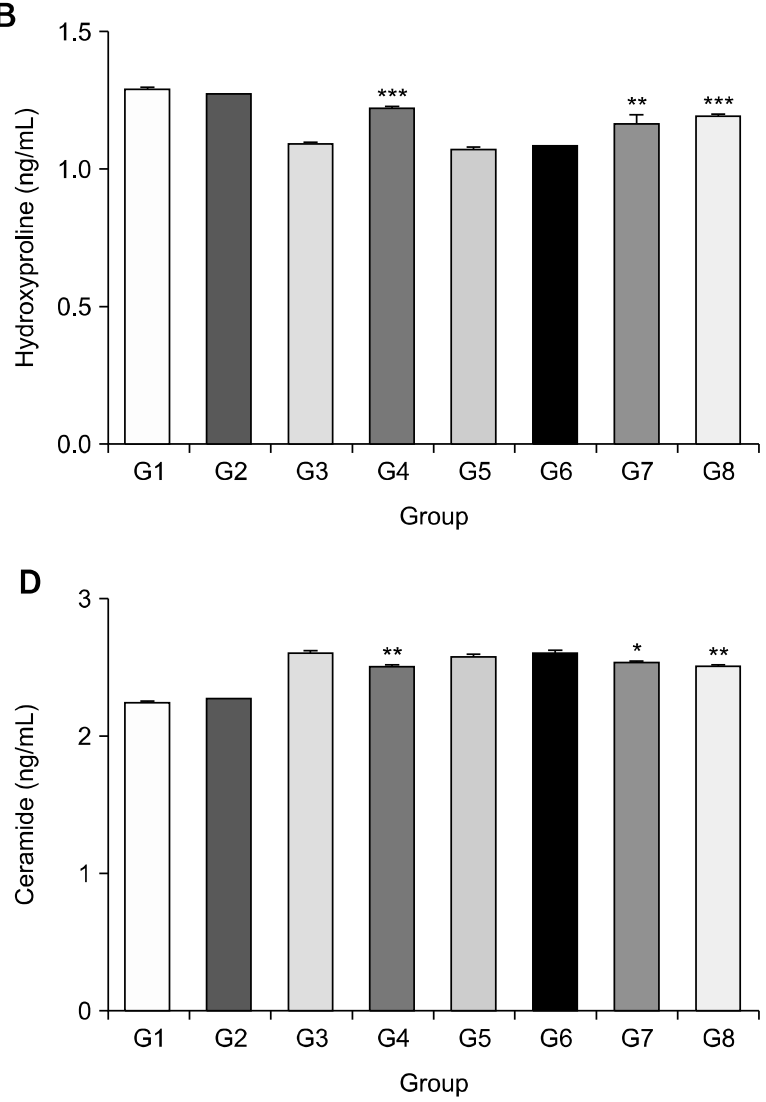

Fig. 8. Effect of test substances on collagenase, hydroxyproline, hyaluronic acid, and ceramide levels. Eight groups (G1 G8) in total were employed for the experiment. G1 was control and G2 was sham control. G3 G8 were induced with ultraviolet-irradiation; G3 was path control (no treatment). G4 was standard drug (hyaluronic acid) treated. G5 (low dose) and G6 (high dose) were treated with test compound A; G7 (low dose) and G8 (high dose) were treated with test compound B. After 42 days of treatment, the levels of collagenase, hydroxyproline, hyaluronic acid, and ceramide levels were measured by ELISA kit. The plasma from the treated and untreated animals was used for analysis as per the kit protocol. The values were expressed in mean \pm SEM. ${ }^{*} P<0.05$, ${ }^{* *} P<0.01$, and ${ }^{* * *} P<0.001$ was considered statistically significant when compared to group 3 values.

The major causes of skin aging are passage of time (intrinsic aging) and cumulative exposure to external influences such as UV radiation and smoking (extrinsic aging) (Yaar and Gilchrest, 2007; Langton et al., 2010). Intrinsically aged skin is characterized by fine wrinkling and reduced elasticity, whereas extrinsically aged skin exposed to UV light is associated with induction of deep wrinkles and significant losses of elasticity (Agache et al., 1980; Warren et al., 1991). Of the external factors that induce skin aging, UV radiation is considered the key cause of skin damage, which is characterized by deep wrinkles, roughness, laxity, and pigmentation (Choi et al., 2010). Many studies have reported that levels of collagen in the skin are decreased significantly after UV radiation (Pienimaki et al., 2001). HA is thought to play important functional roles in healthy skin by controlling the phenotype of epithelial cells. In addition, the HA content governs general functions of the skin, such as water content, turgidity, elasticity, and nutrient diffusion. Detailed information has been previously reported on the regulatory pathways of epidermal HA synthesis (Rilla et al., 2002) the function of epidermal HA (Tammi and Tammi, 1991; Pie- nimaki et al., 2001) and the expression of epidermal HA synthases and HA receptors (Rilla et al., 2002).

Antioxidants, such as carotenoids (lutein and zeaxanthin), diterpenes, flavonoids, vitamins A, C, D, and E, essential omega-3-fatty acids, and some proteins, have been studied for their beneficial anti-aging effects and are used as ingredients in dietary supplements to minimize the increased levels of harmful ROS (Schagen et al., 2012). The effectiveness of lutein and zeaxanthin in helping to protect the skin from damage caused by environmental exposure arises from their antioxidant effects, which has been a subject of research for over a decade (Roberts et al., 2009). Since humans are unable to synthesize these macular carotenoids, dietary supplements help meet the body's requirement (Juturu et al., 2016). In addition, carnosic acid in combination with other bioactive compounds such as lutein and zeaxanthin could be an ideal choice for natural skin protectors.

In conclusion, the present study suggests that chronic UV induces progressive loss of HA from the upper dermis due to downregulating transcription. Furthermore, the proprietary lutein, zeaxanthin, and rosemary formulation 
demonstrates a protective role in these circumstances. The study concluded that the proprietary lutein, zeaxanthin, and rosemary formulation and standard drug HA better protects UV irradiated skin against dehydration.

\section{ACKNOWLEDGEMENTS}

The authors would like to thank the management 182 of Katra Phytochem Private Limited, India, Cosmax Bio, Inc., Korea, and Daehan Chemtech Co., Ltd., Korea, for their support and appreciation of the benefits gained from this research work.

\section{AUTHOR DISCLOSURE STATEMENT}

The authors declare no conflict of interest.

\section{REFERENCES}

Agache PG, Monneur C, Leveque JL, De Rigal J. Mechanical properties and Young's modulus of human skin in vivo. Arch Dermatol Res. 1980. 269:221-232.

Aruoma OI, Halliwell B, Aeschbach R, Löligers J. Antioxidant and pro-oxidant properties of active rosemary constituents: carnosol and carnosic acid. Xenobiotica. 1992. 22:257-268.

Choi HK, Kim DH, Kim JW, Ngadiran S, Sarmidi MR, Park CS. Labisia pumila extract protects skin cells from photoaging caused by UVB irradiation. J Biosci Bioeng. 2010. 109:291-296.

Cooper KD, Oberhelman L, Hamilton TA, Baadsgaard O, Terhune $\mathrm{M}$, LeVee $\mathrm{G}$, et al. UV exposure reduces immunization rates and promotes tolerance to epicutaneous antigens in humans: relationship to dose, CDla-DR + epidermal macrophage induction, and Langerhans cell depletion. Proc Natl Acad Sci USA. 1992. 89:8497-8501.

de Raadt P, Wirtz S, Vos E, Verhagen H. Short review of extracts of rosemary as a food additive. Eur J Nutr Food Saf. 2015. 5: 126-137.

Fisher GJ, Datta SC, Talwar HS, Wang ZQ, Varani J, Kang S, et al. Molecular basis of sun-induced premature skin ageing and retinoid antagonism. Nature. 1996. 379:335-339.

Fisher GJ, Wang ZQ, Datta SC, Varani J, Kang S, Voorhees JJ. Pathophysiology of premature skin aging induced by ultraviolet light. N Engl J Med. 1997. 337:1419-1428.

González-Minero FJ, Bravo-Díaz L, Ayala-Gómez A. Rosmarinus officinalis L. (rosemary): an ancient plant with uses in personal healthcare and cosmetics. Cosmetics. 2020. 7:77. https://doi. org/10.3390/cosmetics7040077

Jia YP, Sun L, Yu HS, Liang LP, Li W, Ding H, et al. The pharmacological effects of lutein and zeaxanthin on visual disorders and cognition diseases. Molecules. 2017. 22:610. https://doi.org/ $10.3390 /$ molecules 22040610

Juturu V, Bowman JP, Deshpande J. Overall skin tone and skinlightening-improving effects with oral supplementation of lutein and zeaxanthin isomers: a double-blind, placebo-controlled clinical trial. Clin Cosmet Investig Dermatol. 2016. 9:325-332.

Kanda N, Watanabe S. Regulatory roles of sex hormones in cutaneous biology and immunology. J Dermatol Sci. 2005. 38:1-7.

Klancnik A, Guzej B, Kolar MH, Abramovic H, Mozina SS. In vitro antimicrobial and antioxidant activity of commercial rosemary extract formulations. J Food Prot. 2009. 72:1744-1752.

Langton AK, Sherratt MJ, Griffiths CE, Watson RE. A new wrinkle on old skin: the role of elastic fibres in skin ageing. Int J Cosmet Sci. 2010. 32:330-339.

Loussouarn M, Krieger-Liszkay A, Svilar L, Bily A, Birtić S, Havaux M. Carnosic acid and carnosol, two major antioxidants of rosemary, act through different mechanisms. Plant Physiol. 2017. 175:1381-1394.

Manuskiatti W, Maibach HI. Hyaluronic acid and skin: wound healing and aging. Int J Dermatol. 1996. 35:539-544.

Margelin D, Medaisko C, Lombard D, Picard J, Fourtanier A. Hyaluronic acid and dermatan sulfate are selectively stimulated by retinoic acid in irradiated and nonirradiated hairless mouse skin. J Invest Dermatol. 1996. 106:505-509.

Mathews-Roth MM, Krinsky NI. Carotenoid dose level and protection against UV-B induced skin tumors. Photochem Photobiol. 1985. 42:35-38.

Nieto G, Ros G, Castillo J. Antioxidant and antimicrobial properties of rosemary (Rosmarinus officinalis, L.): a review. Medicines. 2018. 5:98. https://doi.org/10.3390/medicines5030098

Palombo P, Fabrizi G, Ruocco V, Ruocco E, Fluhr J, Roberts R, et al. Beneficial long-term effects of combined oral/topical antioxidant treatment with the carotenoids lutein and zeaxanthin on human skin: a double-blind, placebo-controlled study. Skin Pharmacol Physiol. 2007. 20:199-210.

Papakonstantinou E, Roth M, Karakiulakis G. Hyaluronic acid: a key molecule in skin aging. Dermatoendocrinol. 2012. 4:253258.

Park M, Han J, Lee CS, Soo BH, Lim KM, Ha H. Carnosic acid, a phenolic diterpene from rosemary, prevents UV-induced expression of matrix metalloproteinases in human skin fibroblasts and keratinocytes. Exp Dermatol. 2013. 22:336-341.

Pienimaki JP, Rilla K, Fulop C, Sironen RK, Karvinen S, Pasonen $S$, et al. Epidermal growth factor activates hyaluronan synthase 2 in epidermal keratinocytes and increases pericellular and intracellular hyaluronan. J Biol Chem. 2001. 276:20428-20435.

Pittayapruek P, Meephansan J, Prapapan O, Komine M, Ohtsuki M. Role of matrix metalloproteinases in photoaging and photocarcinogenesis. Int J Mol Sci. 2016. 17:868. https://doi.org/ 10.3390/ijms 17060868

Quackenbush FW, Miller SL. Composition and analysis of the carotenoids in marigold petals. J Assoc Off Anal Chem. 1972. 55:617-621.

Quan T, Qin Z, Xia W, Shao Y, Voorhees JJ, Fisher GJ. Matrix-degrading metalloproteinases in photoaging. J Investig Dermatol Symp Proc. 2009. 14:20-24.

Ranard KM, Jeon S, Mohn ES, Griffiths JC, Johnson EJ, Erdman JW Jr. Dietary guidance for lutein: consideration for intake recommendations is scientifically supported. Eur J Nutr. 2017. $56: 37-42$.

Rilla K, Lammi MJ, Sironen R, Törrönen K, Luukkonen M, Hascall VC, et al. Changed lamellipodial extension, adhesion plaques and migration in epidermal keratinocytes containing constitutively expressed sense and antisense hyaluronan synthase 2 (Has2) genes. J Cell Sci. 2002. 115:3633-3643.

Roberts RL, Green J, Lewis B. Lutein and zeaxanthin in eye and skin health. Clin Dermatol. 2009. 27:195-201.

Saavalainen K, Pasonen-Seppänen S, Dunlop TW, Tammi R, Tammi MI, Carlberg C. The human hyaluronan synthase 2 gene is a primary retinoic acid and epidermal growth factor responding gene. J Biol Chem. 2005. 280:14636-14644.

Sator PG, Schmidt JB, Rabe T, Zouboulis CC. Skin aging and sex hormones in women - clinical perspectives for intervention by hormone replacement therapy. Exp Dermatol. 2004. 13:3640.

Schagen SK, Zampeli VA, Makrantonaki E, Zouboulis CC. Discovering the link between nutrition and skin aging. Dermatoen- 
docrinol. 2012. 4:298-307.

Sommerburg OG, Siems WG, Hurst JS, Lewis JW, Kliger DS, van Kuijk FJ. Lutein and zeaxanthin are associated with photoreceptors in the human retina. Curr Eye Res. 1999. 19:491-495.

Tammi R, Pasonen-Seppänen S, Kolehmainen E, Tammi M. Hyaluronan synthase induction and hyaluronan accumulation in mouse epidermis following skin injury. J Invest Dermatol. 2005. 124:898-905.

Tammi R, Tammi M. Correlations between hyaluronan and epi- dermal proliferation as studied by $\left[{ }^{3} \mathrm{H}\right]$ glucosamine and $\left[{ }^{3} \mathrm{H}\right]$ thymidine incorporations and staining of hyaluronan on mitotic keratinocytes. Exp Cell Res. 1991. 195:524-527.

Warren R, Gartstein V, Kligman AM, Montagna W, Allendorf RA, Ridder GM. Age, sunlight, and facial skin: a histologic and quantitative study. J Am Acad Dermatol. 1991. 25:751-760.

Yaar M, Gilchrest BA. Photoageing: mechanism, prevention and therapy. Br J Dermatol. 2007. 157:874-887. 